\title{
Determinación de Nitritos por Espectrofotometría UV visible en Productos Embutidos de tipo Jamón
}

Yanina Baca

yanina.baca@unah.edu.hn

Facultad de Ciencias Químicas y Farmacia,

Universidad Nacional Autónoma de Honduras, Tegucigalpa, Honduras

Jhunior Marcía

jmarcia@unag.edu.hn

Facultad de Ciencias Tecnológicas,

Universidad Nacional de Agricultura, Catacamas, Olancho, Honduras

Vilma Chavez

vpchavez@unah.hn

Facultad de Ciencias Químicas y Farmacia,

Universidad Nacional Autónoma de Honduras, Tegucigalpa, Honduras

Sergio Fernández

sfernandez16-0510@unag.edu.hn

Facultad de Ciencias Tecnológicas,

Universidad Nacional de Agricultura, Catacamas, Olancho, Honduras

Hector Montoya

hector.montoya@unah.hn

Facultad de Ciencias Tecnológicas,

Universidad Nacional de Agricultura, Catacamas, Olancho, Honduras

José Baca

jcbaca@unah.hn

Facultad de Ciencias Químicas y Farmacia,

Universidad Nacional Autónoma de Honduras, Tegucigalpa, Honduras

Mauricio Torres

mauromauro232@gmail.com

Facultad de Ciencias Tecnológicas,

Universidad Nacional de Agricultura, Catacamas, Olancho, Honduras

Yaleny Gutierrez

yaleny.gutierrez@unah.hn

Facultad de Ciencias Tecnológicas,

Universidad Nacional de Agricultura, Catacamas, Olancho, Honduras

Rodimiro Padilla

rodimiro.padilla@unah.hn

Facultad de Ciencias Químicas y Farmacia,

Universidad Nacional Autónoma de Honduras, Tegucigalpa, Honduras

Franklin Ore

franklin.ore@unh.edu.pe

Facultad de Ciencias Agrarias, Universidad Nacional de Huancavelica, Perú 


\section{RESUMEN}

El objetivo de esta investigación consistió en cuantificar mediante Espectrofotometría UV-Visible, el contenido de nitritos en productos embutidos de tipo jamón, distribuidos en la zona central de Honduras, para generar una alerta en cuanto a su consumo. Para su alcance, se empleó instrumentos descriptivos tipo encuesta para valorar el nivel de preferencia y la técnica de espectrofotometría a una longitud de onda de $540 \mathrm{~nm}$ para evaluar el contenido de nitritos en tres diferentes tratamientos. Los resultados sugieren que los productos embutidos de mayor aceptación por el consumidor son los de tipo jamón, principalmente aquellos que se elaboran a partir de carne de pollo. De igual manera esta investigación genera una alerta sobre la salud del consumidor, en vista que dos terceras partes de las marcas comerciales empleadas para esta investigación, no cumplen con la normativa de uso de aditivos, según lo establecido por el Codex Alimentarius.

Palabras clave: embutidos, jamón, nitritos, codex alimentarius y espectrofotometría. 


\title{
Determination of Nitrites by UV-visible Spectrophotometry in Products Ham-type Sausages
}

\begin{abstract}
The objective of this research was to quantify, using UV-Visible Spectrometry, the nitrite content in ham-type sausage products, distributed in central Honduras, to generate an alert regarding their consumption. For its scope, descriptive instruments such as a survey were used in consumers of these products, to determine their preference. In turn, the spectrometry technique at a wavelength of $540 \mathrm{~nm}$ was used to evaluate the nitrite content in three different treatments. The results suggest that the sausage products most accepted by the consumer are those of the ham type, mainly those made from chicken meat. Similarly, this research generates an alert on consumer health, given that twothirds of the trademarks used for this research do not comply with the regulations for the use of additives, as established by the Codex Alimentarius.
\end{abstract}

Keywords: sausages, ham, nitrites, codex alimentarius and spectrophotometry.

Artículo recibido: 27 marzo 2021 Aceptado para publicación: 30 abril 2021 Correspondencia: yanina.baca@unah.edu.hn Conflictos de Interés: Ninguna que declarar 


\section{1- INTRODUCCIÓN}

Un aditivo es una sustancia que se añade a los alimentos para mantener o mejorar su inocuidad, su frescura, su sabor, su textura o su aspecto, han sido utilizados desde hace siglos como conservantes principalmente en productos embutidos (OMS, 2018). Los embutidos son productos elaborados a partir de carne, sangre o mezcla de ambas, que además de esencias y hierbas, contiene aditivos que se incorporan con un fin tecnológico o sensorial (Velasquez et al., 2014). El jamón por ejemplo es un producto embutido, en el que se utilizan nitratos y nitritos como aditivos, estos le dan el sabor, olor, textura y color característicos, además funcionan como preservantes, sin embargo, su uso ha sido cuestionado por su potencial toxicidad residual y la posible formación de metahemoglobina, y nitrosaminas. (Gómez, 2006; Chimenos, 2008; Habermeyer \& Eisenbrand, 2019; Kalaycıŏlu \& Erim, 2019).

En cuanto al uso de estos aditivos, la Agencia Federal de Alimentos y Medicamentos (FDA) recomienda no exceder de 200 ppm para nitritos y nitratos (o una combinación de ambos) (Codex, 2019a). Asimismo, el empleo de antioxidantes naturales como el eugenol, sería una alternativa con potencial interés en la industria cárnica para aumentar su durabilidad. (Fuentes et al., 2020; Marcía et al., 2020).

Por lo anterior, el objetivo de esta investigación fue determinar mediante espectrofotometría UV-Visible, el contenido de nitritos en productos embutidos de tipo jamón, distribuidos en la zona central de Honduras, para generar una alerta en cuanto a su consumo.

\section{2- MATERIALES Y METODO}

El método empleado en esta investigación es de enfoque mixto y de orden transversal mediante entrevista y análisis de laboratorio. Para su alcance se seleccionó aleatoriamente a un grupo de 100 personas, pertenecientes al campus de la Universidad Nacional Autónoma de Honduras. Se tomó como criterios de inclusión; edades comprendidas entre 18-25 años y consumidores de productos cárnicos embutidos. Se aplicó una encuesta como instrumento descriptivo para obtener información sobre los principales proveedores de embutidos en la zona urbana de Tegucigalpa, Francisco Morazán, Honduras. Finalmente se tomó muestras al azar de $5 \mathrm{~kg}$ de cada una de las principales marcas comerciales y se trasladaron al Laboratorio de Ciencias de los Alimentos de la UNAH, para el desarrollo de análisis químicos sobre la cuantificación de nitritos en jamón. 
La metodología utilizada para la cuantificación de nitritos se basa en un cambio químico-colorimétrico a una longitud de onda de $540 \mathrm{~nm}$ mediante espectrofotometría de doble haz, mediante espectrofotómetro marca SHIMADZU Uv-1700 pharmaSpec (Manasquan, USA) y celdas de material cuarzo de $1 \mathrm{~cm}$ de diámetro.

\section{Procesamiento de las muestras}

Las muestras se tomaron aleatoriamente en los supermercados de la zona urbana de Tegucigalpa en las cercanías de la UNAH. Posteriormente se trasladaron al Laboratorio Ciencias de los Alimentos en medios herméticos a una temperatura de $10^{\circ} \mathrm{C} \pm 2^{\circ} \mathrm{C}$.

Para la preparación de las muestras, se tomó $1 \mathrm{~kg}$ jamón de cada marca comercial (tratamiento), etiquetándose como $\mathrm{T}_{1}, \mathrm{~T}_{2} \mathrm{y} \mathrm{T}_{3}$. Asimismo, cada tratamiento se segmentó en 10 grupos de 100 g cada uno. El tamizaje se efectuó a partir de muestras aleatorizadas de $100 \mathrm{~g}$ de jamón por tratamiento, se introdujeron en un Erlenmeyer de $250 \mathrm{~mL}$ y se agregó $100 \mathrm{~mL}$ de agua destilada, además, se llevó a digestión durante 2 h en baño María a $80^{\circ} \mathrm{C} \pm 2^{\circ} \mathrm{C}$, añadiendo porciones de $50 \mathrm{~mL}$ de agua destilada cada $30 \mathrm{~min}$ con agitación constante. Posteriormente la muestra se filtró por gravedad mediante un embudo, con la finalidad de eliminar residuos y obtener la solución acuosa. Esta se trasvasó a un matraz de $250 \mathrm{~mL}$ y se aforó con agua destilada.

Para la preparación del estándar de nitrito se empleó 500 mg en 1000 mL de agua destilada, se tomó $1 \mathrm{~mL}$ de la solución y se adicionó $2.5 \mathrm{~mL}$ de SFA (Sulfanilamida) y $2.5 \mathrm{~mL}$ NED (1-Naftiletilendiamina), se aforó con agua destilada en un matraz volumétrico de $100 \mathrm{~mL}$. El blanco se preparó a partir de $20 \mathrm{~mL}$ de agua destilada y las soluciones SFA y NED a las mismas concentraciones que los estándares anteriores. Finalmente, las soluciones estándares se dejaron reposar durante $15 \mathrm{~min}$, para su posterior lectura por triplicado en el espectrofotómetro a una longitud de onda de $540 \mathrm{~nm}$ (Figura 1).

Figura 1. Procedimiento realizado. a. Fraccionamiento y pesado de la muestra. $\boldsymbol{b}$. Digestión de la muestra. c. Filtrado de la muestra. d. Trasvaso de la muestra a un matraz volumétrico. e. Reposo de la muestra por 15 minutos después de agregados los reactivos SFA y NED. f. Lectura de las soluciones en el espectrofotómetro. 


\section{Figura 1.}

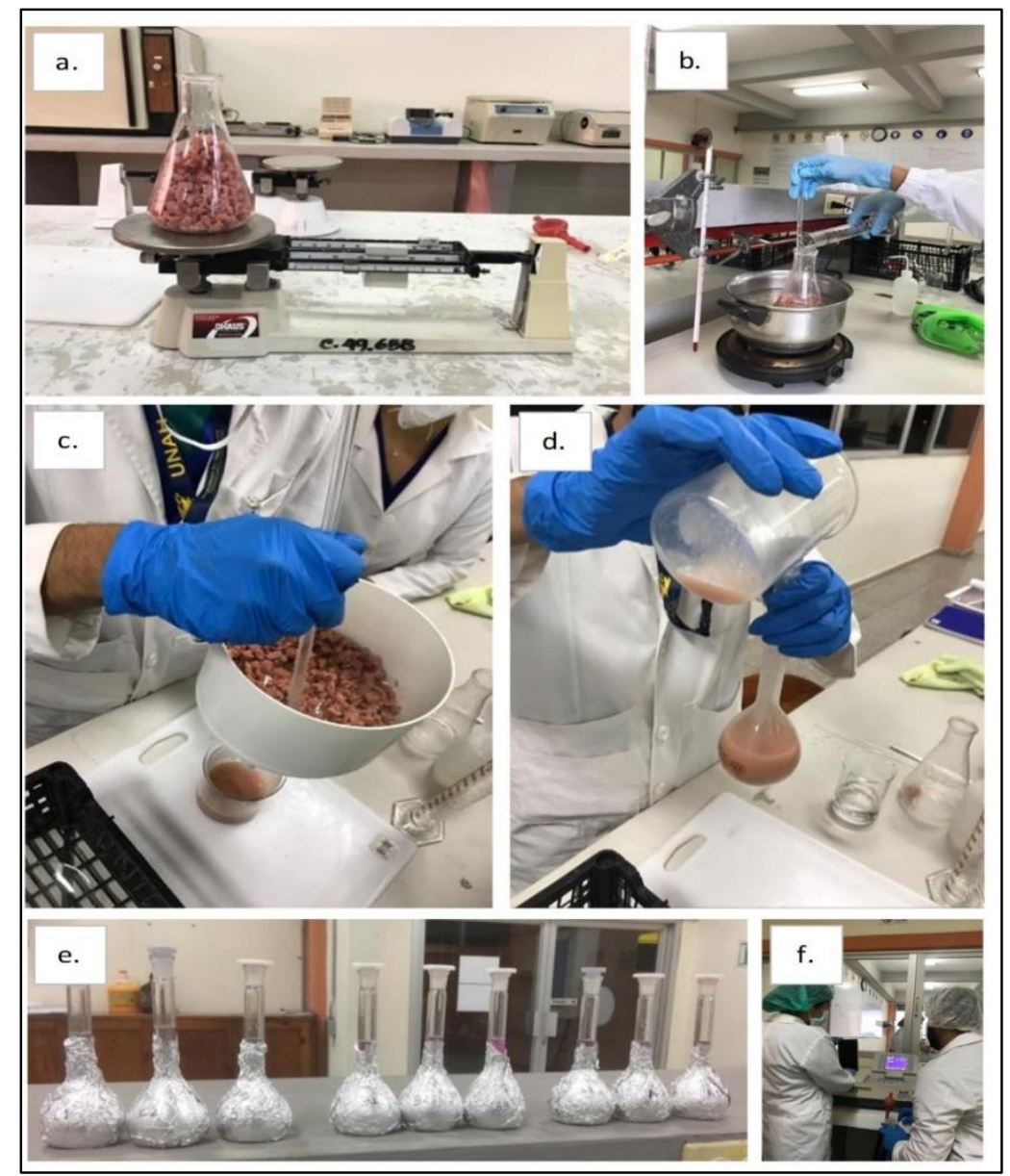

\section{RESULTADOS Y DISCUSIÓN}

A partir de modelos descriptivos tipo encuesta, se determinó que la frecuencia de consumo de productos embutidos oscila entre 2 y 3 veces por semana en la población de la zona central de Tegucigalpa, Francisco Morazán, Honduras (Figura 2). Siendo el jamón el producto embutido de mayor consumo (Tabla 1), principalmente los que emplean en su formulación carne de pollo (Tabla 2). Estos resultados afirman lo expresado por Corzo (2018) y la Organización Mundial de la Salud (2015); quienes expresan que los productos embutidos tienen un efecto adictivo en el consumidor, generando mayor demanda de consumo en comparación con otros tipos de derivados cárnicos. Asimismo, Cruz (2015); relaciona el consumo de estos productos con el desarrollo potencial de enfermedades crónicas como el cáncer. 
Figura 2. Frecuencia de consumo semanal de productos embutidos

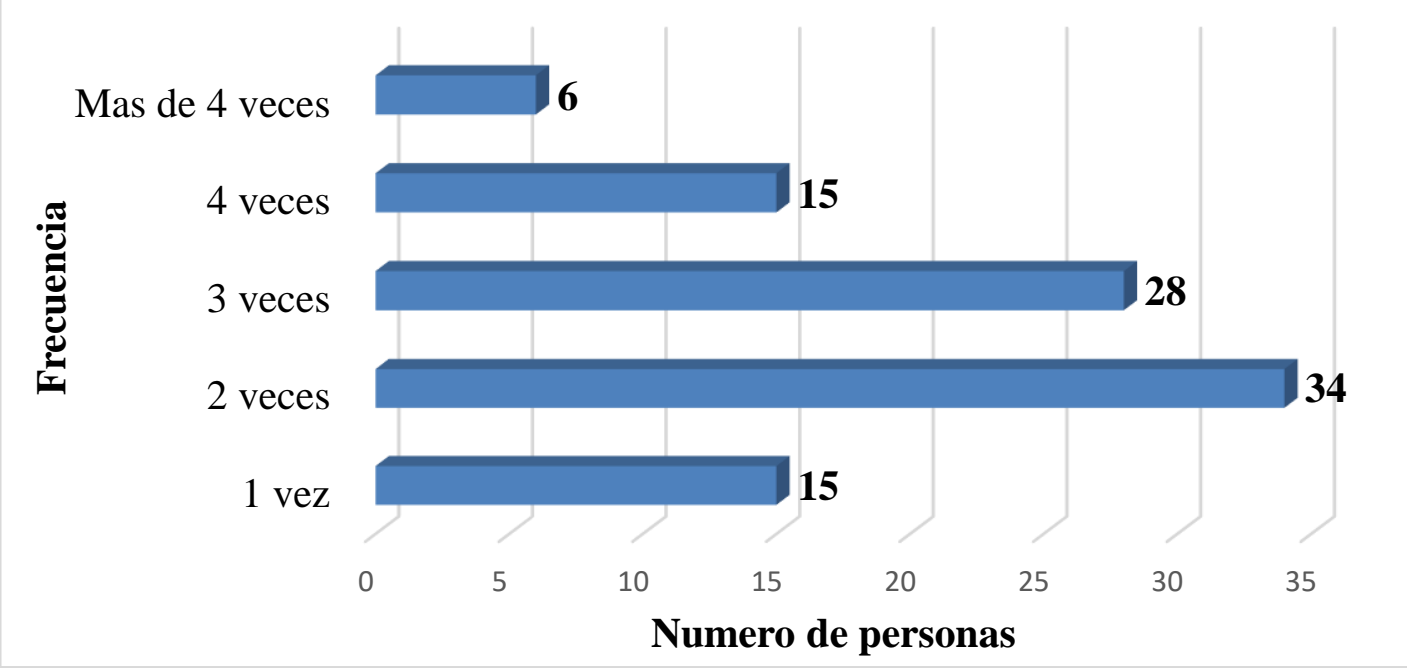

Tabla 1. Porcentaje de consumo de productos embutidos

\begin{tabular}{c|c}
\hline Producto embutido & Porcentaje de consumo (\%) \\
\hline Hot dog & 22.47 \\
\hline Chorizo copetín & 13.56 \\
\hline Jamón & 29.09 \\
\hline Mortadela & 19.38 \\
\hline Chorizo extremeño & 13.55 \\
\hline Otros & 1.94 \\
\hline
\end{tabular}

Tabla 2. Tipo de jamón más consumido

\begin{tabular}{c|c}
\hline Tipo de carne & Porcentaje de consumo (\%) \\
\hline Jamón de cerdo & 37.32 \\
\hline Jamón de res & 18.31 \\
\hline Jamón de pollo & 44.36 \\
\hline
\end{tabular}

La tabla 3, muestra los resultados del contenido de nitritos obtenido entre los tratamientos mediante la técnica de espectrofotometría UV-visible, determinando que dos de cada tres marcas comerciales de embutidos de tipo jamón, que se distribuyen en Tegucigalpa, Francisco Morazán, Honduras, no cumple con la normativa de regulación del Codex Alimentarius. Considerándose su consumo como un potencial riesgo para la salud de esta población. Otros autores como Anton \& Lizaso (2001) y Gaviria \& Montes (2017); expresaron que el uso incorrecto de nitritos como conservantes de alimentos, puede generar intoxicaciones o formación de compuestos carcinogénicos en el ser humano, por lo que su uso debe estar dentro de los parámetros establecidos para el uso de aditivos 
alimentarios (Codex, 2019b).

Tabla 3. Contenido de nitritos entre los tratamientos

\begin{tabular}{c|c|c|c|c}
\hline Tratamiento & ABS & $\begin{array}{c}\text { Concentración } \\
(\mathbf{m g} / \mathbf{k g})\end{array}$ & $\begin{array}{c}\text { Normativa CO- } \\
\mathbf{D E X} \\
\mathbf{m g} / \mathbf{k g}\end{array}$ & Aplicabilidad \\
\hline T1 & $0.489 \pm 0.123$ & $218.836 \pm 86.867$ & 200 & No cumple \\
\hline T2 & $0.124 \pm 0.853$ & $40.906 \pm 28.139$ & 200 & Cumple \\
\hline T3 & $0.796 \pm 0.490$ & $262.050 \pm 16.148$ & 200 & No cumple \\
\hline Blanco & $0.470 \pm 0.336$ & $173.943 \pm 17.183$ & 200 & Cumple \\
\hline
\end{tabular}

*Media \pm desviación estándar.

\section{CONCLUSIÓN}

A partir de espectrofotometría UV-Visible se determinó que la mayor parte de productos embutidos de tipo jamón, que se comercializa en Tegucigalpa, Honduras, no cumplen con la normativa Codex Alimentarius en cuanto al contenido de nitritos empleados como conservante. Asimismo, evidencia que las agencias de regulación sanitarias del país no están monitoreando con frecuencia el uso de estos aditivos. Por lo que esta investigación promueve una alerta en la población hondureña, en cuanto al peligro del consumo de nitritos en productos embutidos y el potencial riesgo que este puede causar en decremento de la salud de sus consumidores.

\section{REFERENCIAS}

Anton, A. \& Lisazo, J. (2001). Nitritos, nitratos y nitrosaminas. Fundación Ibérica para la Seguridad Alimentaria, Madrid, España. 7 pp. Disponible en: https://www.academia.edu/25921074/FUNDACI\%C3\%93N_IB\%C3\%89RICA_P

\section{ARA_LA_SEGURIDAD_ALIMENTARIA}

Chimenos-Küstner, E. (2008). Aspectos prácticos en la prevención del cáncer oral. Avances en odontoestomatología, 24 (1): 61-67. Disponible en: http://scielo.isciii.es/pdf/odonto/v24n1/original5.pdf

Codex Alimentarius. (2019a). Documento de debate sobre el uso de nitratos ( $\sin 251$, 252) y nitritos ( $\sin 249,250)$. Programa conjunto FAO/OMS sobre normas alimentarias. Comité del Codex sobre aditivos alimentarios: 51. ${ }^{a}$ Reunión. Roma, Italia. 29 pp. Disponible en: http://www.fao.org/fao-who-codexalimentarius/sh- 
proxy/es/?lnk=1\&url=https\%253A\%252F\%252Fworkspace.fao.org\%252Fsites\%25 2Fcodex\%252FMeetings\%252FCX-711-51\%252FWD\%252Ffa51_09s.pdf

Codex Alimentarius. (2019b). Norma General para los Aditivos Alimentarios. CODEX STAN 192-1995. 1-9 p. Disponible en: http://www.fao.org/fao-whocodexalimentarius/sh-

proxy/en/?lnk=1\&url=https $\% 253 \mathrm{~A} \% 252 \mathrm{~F} \% 252 \mathrm{Fworkspace}$.fao.org $\% 252 \mathrm{Fsites} \% 2$ 52Fcodex\%252FStandards\%252FCXS\%2B192-1995\%252FCXS_192s.pdf

Corzo-Rosa, N.L. (2018). Evaluación del consumo de embutidos en adolescentes y su aporte de energía, macronutrientes, sodio y aditivos a la dieta. (Tesis de pregrado). Universidad Rafael Landívar, Guatemala. 81 pp. Disponible en: http://recursosbiblio.url.edu.gt/tesisjrcd/2018/09/15/Corzo-Nathalie.pdf

Cruz, J. (2015). La OMS relaciona el consumo de carnes rojas y carnes procesadas con el cáncer. Eurocarne, No. 230: 149-157. Disponible en: http://www.eurocarne.com/boletin/aecoc_prueba_2016/images/24010.pdf

Fuentes, JAM., Fernandez, IM., Aleman, RS., Maldonado, SAS., Roger, LF., Funez, NH., Chavarría, LA. \& Kayanush, A. (2020). Chemical Characterization of the Essential Oil of Syzygium aromaticum and its Antimicrobial Activity Against A Probiotic Lactobacillus Acidophilus. European Scientific Journal ESJ, 16 (15): 33. https://doi.org/10.19044/esj.2020.v16n15p33

Gaviria-Bolivar, E.M. \& Montes-Amador, L.H. (2017). Aplicación de la metodología de evaluación de riesgos en un estudio de caso relacionado con la presencia de nitritos y nitratos en carne. (Tesis de pregrado). Corporación Universitaria Lasallista, Antioquia, Colombia. 83 pp. Disponible en: http://repository.lasallista.edu.co/dspace/bitstream/10567/2264/1/Presencia_nitritos _nitratos_carne.pdf

Gómez-González, M.G. (2006). Identificación y Cuantificación de Nitratos y Nitritos utilizados como aditivos en una marca de jamón tipo popular de alto consumo que expende en supermercados de la ciudad capital. (Tesis de pregrado). Universidad de San Carlos de Guatemala. 70 pp. Recuperado de https://bibliotecafarmacia.usac.edu.gt/Tesis/QF955.pdf

Habermeyer, M., \& Eisenbrand, G. (2019). N-Nitroso Compounds in Foods. En: Varelis, P., Melton, L., Shahidi, F. Encyclopedia of Food Chemistry. Elsevier, p. 593-602. doi:10.1016/b978-0-08-100596-5.21824-6 
Kalaycıoğlu, Z. \& Erim, F.B. (2019). Nitrate and Nitrites in Foods: Worldwide Regional Distribution in View of Their Risks and Benefits. J Agric Food Chem., 67(26):7205-22. Doi: 10.1021/acs.jafc.9b01194

Marcía-Fuentes, J., Torres-Loza, M., Varela-Murillo, I., Chavarría-Carrión, L., Sanabria-Ortega, H., \& Díaz-Antúnez, H. (2020). Efecto del eugenol en la vida util de una salsa de chile jalapeño (Capsicum annum) y papaya (Carica papaya). Nexo Revista Científica, 33(01): 69-76. https://doi.org/10.5377/nexo.v33i01.10046

Organización Mundial de la Salud (OMS). (2018). Aditivos alimentarios. Recuperado de https://www.who.int/es/news-room/fact-sheets/detail/food-additives

Organización Mundial de la Salud (OMS). (2015). Carcinogenicidad del consumo de carne roja $\mathrm{y}$ de la carne procesada. Disponible en: https://www.who.int/features/qa/cancer-red-meat/es/

Vargas-Velásquez, C., López-Reinoso, A.R. \& Flores-Artunduaga L.M.B. (2014). Evaluación de la concentración de nitratos/nitritos y cloruro de sodio en embutidos expendidos en la ciudad de Tarija. Revista Ventana científica, 1(7): 1-8. Recuperado de: http://www.revistasbolivianas.org.bo/pdf/rvc/v1n7/v1n7_a02.pdf 